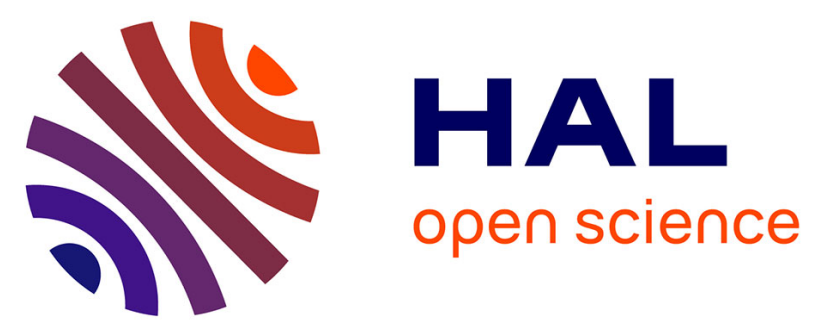

\title{
Éducation thérapeutique et responsabilité juridique des équipes soignantes dans le cadre de la loi Hôpital, Patient, Santé, Territoire de juillet 2009, Du devoir du médecin au droit du patient
}

\author{
Anne-Sophie Baillet, Rémi Gagnayre, Vincent de Andrade, Jean-François \\ d'Ivernois, Isabelle Poirot Mazères
}

\section{To cite this version:}

Anne-Sophie Baillet, Rémi Gagnayre, Vincent de Andrade, Jean-François d'Ivernois, Isabelle Poirot Mazères. Éducation thérapeutique et responsabilité juridique des équipes soignantes dans le cadre de la loi Hôpital, Patient, Santé, Territoire de juillet 2009, Du devoir du médecin au droit du patient. Educ Ther Patient/Ther Patient Educ, 2011, 3 (2), pp.S207 - S215. 10.1051/tpe/2011109 . hal-01556758

\section{HAL Id: hal-01556758 \\ https://sorbonne-paris-nord.hal.science/hal-01556758}

Submitted on 7 Jul 2017

HAL is a multi-disciplinary open access archive for the deposit and dissemination of scientific research documents, whether they are published or not. The documents may come from teaching and research institutions in France or abroad, or from public or private research centers.
L'archive ouverte pluridisciplinaire HAL, est destinée au dépôt et à la diffusion de documents scientifiques de niveau recherche, publiés ou non, émanant des établissements d'enseignement et de recherche français ou étrangers, des laboratoires publics ou privés. 


\title{
Article original/Original article
}

\section{Éducation thérapeutique et responsabilité juridique des équipes soignantes dans le cadre de la loi Hôpital, Patient, Santé, Territoire de juillet 2009, Du devoir du médecin au droit du patient ${ }^{\star}$}

\author{
Anne-Sophie Baillet ${ }^{1 \star \star}$, Rémi Gagnayre ${ }^{1}$, Vincent de Andrade ${ }^{1}$, Jean-François d'Ivernois ${ }^{1}$, \\ Isabelle Poirot Mazères ${ }^{2}$ \\ ${ }^{1}$ Laboratoire de Pédagogie de la Santé EA 34-12, Université Paris13-Bobigny, Bobigny, France \\ ${ }^{2}$ Laboratoire TACIP-Institut Maurice Hauriou, Université Toulouse 1-Capitole, Toulouse, France
}

(Reçu le 19 avril 2011, accepté le 23 juin 2011)

\begin{abstract}
Résumé - Introduction : L'éducation thérapeutique du patient (ETP) connaît un développement important ces dernières années. Description : C'est ici sous l'angle juridique que nous aborderons cette pratique. Deux points attireront notre attention. Le premier concerne à la fois la responsabilité juridique des soignants et celle des «patients experts ». Les principaux motifs susceptibles d'engager la responsabilité juridique des équipes sont ainsi la violation du secret professionnel, le manquement au devoir d'information et la faute d'imprudence. Le second point portera sur la confidentialité. Cette dernière revêt une importance particulière puisque le groupe de patients est amené à se confier publiquement sur des sujets médicaux et intimes. Il est nécessaire que l'obligation de discrétion soit respectée non seulement de la part des professionnels - y compris par les «patients experts » - mais aussi par les patients au sein du groupe lui même. Discussion : On conseillera notamment la mise en place d'une charte de confidentialité ou dans le document de consentement signé par le patient en intégrant le programme - valeur contractuelle sur le plan juridique - précisant le respect de cette obligation. Conclusion : Cette analyse postule que l'ETP réactive des notions juridiques susceptibles de faire jurisprudence dans les années à venir, dont les équipes soignantes doivent avoir conscience. Cette «lucidité juridique » des pratiques en ETP doit ainsi permettre d'en augmenter la qualité.
\end{abstract}

Mots clés : éducation thérapeutique du patient / loi HPST / responsabilité juridique / confidentialité

\begin{abstract}
The patient therapeutic education and juridical responsibility for medical team, in the law HPST, July 2009: The duty doctor in the right of the patient. Introduction: For several years, the therapeutic patient education (TPE) has raised in an important way. Description: We will study here this issue on a juridical meaning. We have to point out two major facts. The first one speaks about juridical responsibility for both doctors and nurses team and also "expert patient". Motivations to engage a team juridical responsibility are violation of professional secrecy, lack of information duty and carelessness. The second issue concerns confidentiality. This last one represents a specific issue because a patient group is led itself to confide some medical or personal subjects. The discretion obligation needs to be respected not only by professional team (including "expert patients") but also by the patient inside the group itself. Discussion: A confidential commitment or a consent note signed up by the patient, including the whole plan, contractual meaning on a legal issue - has to be set up. Conclusion: This analysis postulate PTE creates in the future years a precedent due to legal issues for which the medical teams have to be aware. We must be aware that this legal clearness in matter of PTE practice could allow to improve it quality.
\end{abstract}

Key words: therapeutic patient education / law HPST / juridical responsibility / confidentiality

\footnotetext{
^ Article issu du mémoire de Master 2 Droit de la Santé, Université Toulouse Capitole.

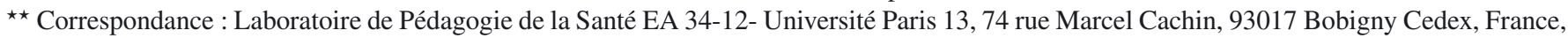
annesophiebaillet@hotmail.fr
} 
Abbreviations: ETP : Éducation Thérapeutique du Patient, Loi HPST : Loi Hôpital, Patient, Santé, Territoire, HAS : Haute Autorité de Santé, INPES : Institut National de Prévention et d'Éducation pour la Santé, CSP : Code de Santé Publique, ARS : Agence Régionale de Santé.

\section{Introduction}

L'éducation thérapeutique du patient (ETP) connaît depuis une vingtaine d'années un fort développement en France. L'inclure dans les actes de soin comporte des bénéfices multiples. De nombreux rapports et études l'ont montré. La Haute Autorité de Santé (HAS) a notamment proposé, en 2007, des recommandations sur cette pratique [1]. L'enquête EDUPEF [2] menée par l'Institut national de prévention et d'éducation pour la santé (INPES) a en outre identifié et décrit les pratiques d'ETP avec leurs modalités d'organisation dans les établissements de santé français, faisant le constat d'une implantation significative de l'éducation thérapeutique en 2008 et favorisant sa reconnaissance par les pouvoirs publics en 2009 par les articles L1161-1 [3] et suivants du code de santé publique. Cette reconnaissance par la loi et l'application dans le champ de la médecine réactive l'arsenal juridique sur d'une part la relation soignant-soigné, en mettant à la charge des professionnels de santé des obligations et non plus seulement des devoirs; d'autre part, la reconnaissance d'un nouvel acteur comme le patient «expert», qui nécessite un cadre juridique et interroge les pratiques. Au moment même où des programmes d'ETP sont autorisés, il semble utile pour les équipes soignantes d'explorer plus précisément la responsabilité juridique de l'ensemble des acteurs.

\section{Développement d'une nouvelle pratique en santé : l'éducation thérapeutique du patient}

"L'éducation thérapeutique du patient [ETP] devrait permettre aux patients d'acquérir et de conserver les capacités et les compétences qui les aident à vivre de manière optimale leur vie avec leur maladie. Il s'agit donc d'un processus permanent, intégré dans les soins et centré sur le patient» [4]. C'est ainsi qu'elle a été définie et reconnue en 1998 par l'OMS-Europe. Une définition reprise en 2007 par la HAS et l'INPES [1] dans le cadre de recommandations lui conférant une légitimité, même s'il s'agit à l'époque d'un cadre non normatif.

\subsection{Le patient, véritable acteur de sa santé}

L'éducation thérapeutique du patient s'adresse en majorité à des personnes souffrant de pathologies chroniques (soit 15 à 20 millions de personnes en France). Le caractère de longue durée de la pathologie et l'impossibilité pour le monde médical d'être présent de manière continue imposent de trouver de nouvelles formes de prises en charge [5], notamment en déléguant aux patients certaines compétences que les soignants ne peuvent plus assurer directement auprès d'eux. Ils deviennent alors de véritables acteurs de leur santé dans le cadre d'un partenariat soignant-soigné. L'autonomie s'opère par un apprentissage significatif du patient afin qu'il puisse mobiliser des compétences pour gérer sa maladie et son traitement et s'assurer une meilleure qualité de vie [6]. Pour l'ensemble de la communauté médicale, les sociétés savantes et les pouvoirs publics, il semble désormais acquis que le curatif et le préventif ne sont pas des concepts opposés mais complémentaires : "L'éducation thérapeutique du patient apparaît comme une passerelle possible, puisque cette pratique allie les impératifs du curatif et les exigences du préventif dans une même unité de temps et de lieu» [6]; elle est curative par l'intégration à l'acte de soins, mais aussi préventive car elle vise à l'information, l'apprentissage du patient, dans le but de lui permettre de limiter les incidents, de retarder les complications. Ainsi l'éducation thérapeutique contribue-t-elle au renforcement d'une prévention secondaire et tertiaire [7].

\subsection{Développer et renforcer une politique de prévention}

Pour les pouvoirs publics, l'éducation thérapeutique représente l'opportunité de développer et renforcer une politique de prévention [6] qui, initiée dès les années 1970-1980, a fait l'objet d'une reconnaissance institutionnelle progressive dans le système français :

- par la loi du 4 mars 2002 relative au droit des malades et à la qualité du système de santé avec la création de l'INPES, désormais principal acteur du système de prévention en France;

- par la loi du 9 août 2004 relative à la politique de santé publique qui a reconnu à la prévention un caractère prioritaire, notamment à travers cinq plans nationaux, dont un portant sur l'amélioration de la qualité de vie des personnes atteintes de maladies chroniques pour la période 2007 2011.

\subsection{Modification de la relation soignant-soigné}

Autre facteur déterminant dans le développement de l'ETP en France : la modification de la relation soignant-soigné. Au principe de bienfaisance qui a conduit au paternalisme, on oppose aujourd'hui les suivants : primat de l'autonomie de la personne; préservation des capacités de choix; necessité de recueillir un consentement éclairé. Auparavant, le patient devait faire entièrement confiance aux médecins, seuls détenteurs du savoir. Des termes comme «prescription» ou « ordonnance» en sont une parfaite illustration. La notion de «privilège thérapeutique», qui reconnaissait la possibilité de dissimuler des 
informations aux patients, figure également dans le code de déontologie de 1955. Une disposition encore d'actualité puisque d'après l'article 35 de ce dernier, le médecin peut dissimuler un diagnostic grave "pour des raisons qu'il apprécie en conscience ».

Un des éléments essentiels expliquant la transition d'une relation paternaliste à un état d'autonomie est l'émergence de la notion de sujet auto-déterminé. Le patient est un individu reconnu dans sa singularité, capable de décider ce qui lui est préférable. Ce nouveau statut a comme conséquence de prendre en compte le point de vue des patients dans les décisions concernant leurs soins. Cependant ce statut croise d'autres logiques comme le consumérisme médical. C'est ainsi qu'on sollicite la satisfaction des patients comme on le fait pour des clients. Les patients qui, selon une logique de sujets auto-déterminés, exprimaient un point de vue régulateur de l'offre de soins sont «approchés » comme des clients d'un service de téléphonie, bancaire. Ainsi, la prestation médicale est désormais qualifiée de prestation de services et se trouve ainsi soumise au respect du droit de la consommation. Concernant le statut du patient, la cour de cassation va expressément qualifier le patient de consommateur de soins $-c f$. l'arrêt du 15 mai 1984 [8]. Elle admet de ce fait l'application des dispositions du droit de la concurrence dans un souci de protection du patient. Le droit français va dans la même direction que le droit communautaire qui a qualifié l'acte médical de prestation de service - $c f$. l'arrêt de la Cour de justice de la Communauté européenne du 31 janvier 1984 [9].

En France, la loi du 4 mars 2002 met fin au paternalisme médical. Elle a pour ambition de rééquilibrer la relation soignant-soigné en instaurant plus de transparence et en renforçant le droit à l'information. Le patient est désormais considéré comme un acteur à part entière de la relation médicale et ne doit plus être un simple usager mais un patient-citoyen; on parle désormais de démocratie sanitaire. Elle désigne l'entrée des usagers en tant qu'acteurs dans le champ de la santé. Le patient doit pouvoir exprimer ses besoins pour pouvoir en discuter et décider.

La loi a donc permis au patient de passer d'un statut de patient relativement passif à celui d'acteur de ses soins. L'éducation thérapeutique s'inscrit dans cette nouvelle perspective. «Ces apprentissages s'inscrivent dans une recherche permanente d'équilibre, dans une négociation entre une norme thérapeutique proposée par le milieu médical et soignant et celle du patient issue de ses représentations, de ses projets, de son système de valeurs et de ses habitudes de vie » [6]. Il y a donc là une volonté manifeste d'éclairer voire de responsabiliser le patient sur sa pathologie en favorisant l'acquisition d'une certaine autonomie.

\section{L'intégration de l'éducation thérapeutique du patient dans le champ juridique}

En France, nous assistons depuis une dizaine d'années à l'intégration progressive de l'éducation thérapeutique dans le champ législatif et politique. Ce n'est qu'en 2001 que la direction générale de la Santé a remis un rapport sur l'ETP, en reconnaissant l'accès à un programme structuré à tous les patients dont l'état de santé le nécessite. Par la suite, les décideurs ont intégré cette pratique dans leur politique de santé. De manière non exhaustive, nous pouvons ainsi citer :

- Le plan d'amélioration de la qualité de vie des personnes atteintes de maladies chroniques pour la période de 2007 2011. Ce plan met l'accent sur l'ETP dans son second axe relatif à l'élargissement de la médecine de soins à la prévention.

- Les rapports de la HAS et de l'INPES, en juin 2007 [1]. Le document de la Haute Autorité de Santé fournit un guide méthodologique concernant la définition de l'ETP, ses finalités, la description de la démarche, les propositions d'évaluation. Il s'agit d'un référentiel complet permettant sa mise en place.

- En matière législative, la loi de mars 2002 relative aux droits des malades et à la qualité du système de santé. Son chapitre 7 portant sur la prévention et l'éducation pour la santé, l'ETP fait désormais partie intégrante de la politique de santé (CSP, art. 1417-1). La loi du 9 août 2004 va reconnaître pour la première fois la responsabilité de l'Etat en matière de santé publique. Cinq plans nationaux ont été ainsi définis, dont un sur la qualité de vie des personnes atteintes de maladies chroniques.

- Enfin, la loi Hôpital, Patient, Santé, Territoire du 21 juillet 2009 modifie en profondeur l'organisation du système de soins en France. La principale disposition est sans doute la création des Agences Régionales de Santé qui auront pour mission de structurer l'ensemble des soins, à la fois hospitaliers et ambulatoires. Elle met l'accent sur une vision globale de l'offre de soins et des problèmes de santé. Si cette loi a pu être contestée, à tort ou à raison sur de nombreux points, la reconnaissance de l'éducation thérapeutique fait largement consensus.

L'ETP est définie à l'article L1161-1 et suivants du code de santé publique, comme «s'inscrivant dans le parcours de soins du patient. Elle a pour objectif de rendre le patient plus autonome en facilitant son adhésion aux traitements prescrits et en améliorant sa qualité de vie. Elle n'est pas opposable aux malades et ne peut conditionner le taux de remboursement de ses actes et des médicaments afférents à sa maladie.»

L'ETP est considérée comme un élément de parcours de soins distinct de l'accompagnement du malade, qui lui a pour objet d'apporter une assistance et un soutien aux malades, à leur entourage, dans la prise en charge de la maladie. L'autorisation des programmes est effectuée par les ARS au niveau territorial en respectant un cahier des charges. Un guide d'évaluation destiné aux ARS a été conçu par la HAS durant l'été 2010 pour les aider dans leur analyse des projets déposés par les équipes soignantes et soumis à autorisation.

La loi HPST reprend bon nombre de points précédemment cités, comme la recherche d'une certaine autonomie pour le patient, l'amélioration de sa qualité de vie, l'observance 
thérapeutique... Elle s'inspire clairement du référentiel élaboré par la HAS/INPES de 2007.

La France joue un rôle précurseur en la matière qui consacre l'ETP comme une pratique à part entière [10], sans l'englober dans les pratiques de promotion et d'éducation pour la santé, à l'inverse des pays voisins comme la Belgique, la Suisse et la Grande-Bretagne [12]. Désormais l'ETP jouit d'une reconnaissance institutionnelle et législative, qui confère à ceux qui la pratiquent des droits et des obligations, source de responsabilités.

\section{L'interprétation de l' ETP dans le cadre général de la relation soignant-soigné}

Les droits des patients constituent le reflet des obligations des médecins; ils se sont construits à travers différentes sources comme le code de déontologie médicale, de nombreuses lois, et des conventions nationales... Ils s'appuient désormais de plus en plus sur la législation. Le curseur s'est déplacé des devoirs du médecin (avec le code déontologie médicale) vers les droits du patient (loi du 4 mars 2002). Ces obligations s'imposent à tous les stades de la prise en charge : prévention, diagnostic et soins.

Plusieurs droits semblent particulièrement intéressants en raison de leur importance dans la pratique de l'ETP. Ils sont issus de grands principes comme la liberté individuelle, la dignité de la personne humaine et enfin la protection de la santé. La loi Kouchner de 2002 a consacré l'émergence d'un nouveau type de relation entre patient et médecin, « la relation équilibrée». Depuis peu, les échecs sont de plus en plus mal tolérés et les médecins se trouvent plus facilement accusés de négligence, voire d'incompétence. En raison de cette « judiciarisation » des rapports entre médecins et patients, l'information du patient et le recueil de son consentement sont devenus une nécessité.

\subsection{L'information}

Ce droit à l'information permet de rétablir une certaine égalité entre celui qui a la connaissance (le médecin) et celui qui ne l'a pas (le patient). La nature de l'information a été précisée par le code de déontologie de 1995 : «...le médecin doit une information claire, loyale et appropriée...Pour des raisons légitimes qu'il apprécie en conscience un malade peut être tenu dans l'ignorance d'un diagnostic grave. » $\mathrm{Ce}$ droit a été consacré en 2002 aux articles 1112-1 et suivants du code de santé publique : "Toute personne a le droit de recevoir une information sur son état de santé », il ne s'agit plus seulement d'un devoir pour le médecin. L'information doit être délivrée préalablement à l'acte de soins ou à la prise en charge du patient, et adaptée à ce dernier, supposant un dialogue afin de vérifier la bonne compréhension du patient. En cas de violation de cette obligation, la charge de la preuve pèse sur le praticien depuis l'arrêt du 25 février 1997 ( $1^{\text {re }}$ chambre civile, 25 juin 1996, Bull. $n^{\circ} 268$, p.188). Cette obligation peut être rapportée par tous moyens même si l'écrit reste la forme la plus sûre. Lorsque le praticien se trouve dans l'impossibilité de prouver l'information, il engage sa responsabilité civile mais non pénale. Il devra réparer le préjudice subi par le patient qui, s'il avait été mieux informé, aurait pu échapper au dommage. C'est la perte de chance qui sera indemnisée.

\subsection{Le consentement}

Cette obligation d'information permet au patient d'accorder ou de refuser de donner son consentement. Ce dernier a fait l'objet d'une évolution dans le code de déontologie médicale traduisant l'évolution de nos sociétés, passant d'une décision prise par le médecin (code de déontologie médicale 1947) à la nécessité de le rechercher dans tous les cas (CDM 1995). Ce principe sera consacré dans le droit positif en 1994 à l'article 16-3 du code civil en mentionnant que le consentement de la personne doit être recueilli au préalable sauf en cas d'urgence, d'incapacité et d'hospitalisation sous contrainte. Avec la loi de 2002, une étape supplémentaire est franchie, qui instaure une véritable codécision : "Toute personne prend avec les professionnels de santé, compte tenu des informations et des préconisations fournies, les décisions concernant sa santé. » (CSP, art. L111-4.) On reconnaît au malade non seulement le droit de consentir (comme cela existait depuis 1947 avec le Code de Nuremberg) mais aussi celui de décider. Ce consentement doit être libre et éclairé, il n'y a aucune forme particulière à respecter, il découle de l'information.

La mise en œuvre de ces deux obligations nécessite, en contrepartie, le respect du secret professionnel. Le $\mathrm{P}^{r}$ Louis Porte, président du conseil national de l'Ordre des médecins, déclarait en 1950 : «Il n'y a pas de médecine sans confiance, de confiance sans confidence et de confidence sans secret. » La loi du 4 mars 2002 l'a intégré à l'article L1110-4 du code de santé publique. Ce devoir traditionnel, pierre angulaire de l'exercice médical, se conjugue aujourd'hui avec le droit à l'information et à la transparence.

\subsection{Le secret professionnel}

Du secret médical, nous sommes passés, en 1994, au secret professionnel qui s'impose à de nombreuses professions. Devoir commun, le secret oblige celui qui a reçu la confidence à se taire dans le but de garantir les droits de la personne. Il en découle que la violation devient effective dès la moindre révélation, en l'absence de toute intention de nuire, dès lors qu'elle est spontanée. Le secret couvre tout ce qui est venu à la connaissance du médecin et du professionnel de santé dans l'exercice de sa profession, c'est-à-dire non seulement ce qui lui a été confié mais aussi ce qu'il a vu ou entendu.

En matière pénale, l'évolution est significative, passant de l'article 378 du code pénal à l'article 226-13. On fait désormais référence au secret professionnel et non plus simplement au secret médical : "La révélation d'une information à caractère secret par une personne qui en est dépositaire soit par 
état, soit par profession, soit en raison d'une fonction ou d'une mission temporaire, est punie d'un an d'emprisonnement et de 15000 euros d'amende. »

Le code de santé publique le mentionne depuis 2002 : «Toute personne prise en charge par un professionnel, un établissement, un réseau de santé ou tout autre organisme participant à la prévention et aux soins a droit au respect de sa vie privée et du secret des informations la concernant [...]. Deux ou plusieurs professionnels de santé peuvent toutefois, saufopposition de la personne dûment avertie, échanger des informations relatives à une même personne prise en charge, afin d'assurer la continuité des soins ou de déterminer la meilleure prise en charge sanitaire possible. Lorsque la personne est prise en charge par une équipe de soins dans un établissement de santé, les informations la concernant sont réputées confiées par le malade à l'ensemble de l'équipe. » (Art. 1110-4.)

Tous les professionnels de santé sont donc soumis au secret professionnel : médecins, infirmières, dentistes, internes, stagiaires...mais aussi secrétaires, personnels administratifs, éducateurs, etc.

Le secret médical est inviolable, mais force est de constater que certaines exceptions lui ont été apportées sous la dénomination de « secret médical partagé » (cf. CSP, art. L1110-4, alinéa 3). Ce secret partagé est toléré dans le cadre d'une collaboration diagnostique ou thérapeutique concernant le patient. Il permet d'échanger des informations entre professionnels de santé et doit se limiter aux données médicales, objectives et non excessives.

Le rappel de ces principes constants du droit de la santé permet de s'interroger sur leur application effective dans les pratiques d'éducation thérapeutique.

\section{La problématique de la confidentialité en éducation thérapeutique}

Actuellement, tout reste à faire dans ce domaine puisque la nouvelle loi de santé publique est récente. Les demandes d'autorisation de programme d'éducation thérapeutique doivent être adressées auprès du directeur de l'agence régionale de santé (cf. CSP, art. L1661-4). Parmi les critères à respecter, l'un porte sur la participation des patients à la conception et à l'animation des programmes d'éducation thérapeutique. La question de la confidentialité va se poser de facto non seulement en raison de la présence de patient en tant qu'éducateur mais aussi en tant que pratique de soins. Rappelons que celleci est fondée sur des notions telles que l'interdisciplinarité des professionnels et sur les échanges entre patients, notamment dans le cadre de séances collectives d'ETP où des éléments très intimes vont être révélés, nécessitant de poser un cadre juridique.

\subsection{Séances d'ETP collectives menées exclusivement par des professionnels de santé}

La confidentialité revêt une importance particulière puisque le groupe de patients est amené à se confier publiquement sur des sujets médicaux et intimes. Il est nécessaire que l'obligation de discrétion soit respectée de part des professionnels mais aussi par les pairs.

Les professionnels sont soumis à différentes obligations :

- Ils sont tenus au respect du secret professionnel sur les échanges intervenus avec les patients (cf. CSP, art. 1110-4).

- L'échange d'informations est possible en raison du secret médical partagé, après information du patient qui n'a pas manifesté son opposition. Le consentement de la personne n'est pas requis en tant que tel, mais il convient de s'assurer de l'absence d'opposition. Sur le terrain, il est souvent constaté l'absence d'une telle demande au patient. Tous les professionnels membres de l'équipe pourront avoir accès au dossier thérapeutique du patient, qu'il soit indépendant du dossier médical ou au contraire inclus. Dans le cas où il serait informatisé, il est plus facile de contrôler l'accès à certaines données; le cas échéant, l'exploitation des données individuelles donnera lieu à une demande d'autorisation auprès de la CNIL (loi du 6 janvier 1978 relative à l'informatique et aux libertés).

- Enfin, le patient qui entre dans un programme doit être informé de l'obligation de discrétion à la charge des professionnels de santé, qui incombe de même au groupe de patients.

Cette information pourra être délivrée de plusieurs manières :

- soit dans le document de consentement (valeur contractuelle sur le plan juridique) signé par le patient en intégrant le programme - qui pourrait contenir tous les principes généraux de l'ETP avec notamment la mention sur la confidentialité patients-professionnels et entre pairs, de manière à ce tout engagement se fasse en connaissance de cause ;

- soit dans une charte relative au fonctionnement d'un programme d'ETP avec les droits et obligations de chaque partie.

\subsection{L'apparition du concept de « patient expert » en éducation thérapeutique}

Depuis quelques années, un nouveau concept se fait jour en éducation thérapeutique : le «patient expert»-aucune terminologie spécifique n'a encore été retenue pour le qualifier. Relativement nouveau en France, ce phénomène est apparu aux USA et en Grande Bretagne [11]. Il s'agit en réalité de mettre l'accent sur une plus grande participation des patients. L'idée qu'ils puissent être considérés comme experts de leur pathologie vient du constat selon lequel beaucoup d'entre eux possèdent des compétences sur leur maladie qui, si elles différent de celles de leur médecin, ne sont pas à négliger pour autant.

L'expertise s'entend différemment dans le système de santé français. Elle est limitée dans l'hypothèse défendue notamment par G. Reach qui précise que le patient n'est expert que de sa propre maladie et ne peut donc être considéré comme «patient éducateur», car «le risque est grand que 
le patient éducateur soit tenté de présenter les résultats de sa propre démarche comme universels, extrapolables au plus grand nombre, alors qu'ils ne sont, stricto sensu, applicables qu'à lui-même dans sa situation médicale et thérapeutique propre » [12]. Cette limite n'empêche pas des associations comme l'Association française des diabétiques (AFD) ou l'Association française des hémophiles (AFH) de mener le projet de former des «patients experts» (ou «patients ressources » tels que les nomme l'AFH [13]). Dans cet exemple, les patients ressources co-animent avec les soignants des séances d'éducation thérapeutique dans le but de favoriser la participation de leurs pairs et leur acquisition de certaines compétences d'autosoins ou d'adaptation à la maladie.

Même en l'absence de données plus tangibles permettant de préciser les caractéristiques du «patient expert», les apports de «patients ressources » dans les programmes d'ETP, il semble que pour les pouvoirs publics ce statut paraît désormais acquis, comme en témoigne la prise en compte de l'intervention des patients dans le guide méthodologique HAS/INPES. Leurs concours peuvent s'inscrire dans le cadre de l'initiative, de la conception, de la mise en œuvre ou encore de l'évaluation d'un programme d'éducation thérapeutique. Pour l'heure, la loi HPST ne mentionne pas l'intervention de «patient expert » mais les critères d'autorisation de programmes d'éducation thérapeutique mentionnent explicitement la participation de patients ou représentants d'associations de santé dans leur mise en œuvre.

Ainsi la reconnaissance de l'ETP et l'implication des patients dans la mise en œuvre de l'éducation thérapeutique posent-elles de nombreuses questions méthodologiques : quels sont les rôles des patients qui interviennent dans ces programmes? Quelle est leur place? Et, quelles que soient les réponses et dans la mesure où leur implication sera croissante, quelles seront les conséquences éventuelles sur le plan juridique de leur participation en tant que professionnels nonsoignants?

\subsection{Séances d'ETP collectives menées à la fois par des professionnels de santé et par des non-soignants}

Les patients vont pouvoir intervenir en tant que partenaires des professionnels de santé en vue notamment d'enrichir l'éducation dispensée. Il peut s'agir de membres associatifs, intervenant à titre bénévole dans un établissement de santé ou bien d'une personne atteinte par la pathologie concernée. Dans le second cas, il s'agit d'un patient nommé expert pour les besoins de notre argumentation. La question est de savoir si ces personnes sont soumises aux mêmes obligations que les soignants.

- Le secret professionnel. Il "couvre l'ensemble des informations concernant la personne venue à la connaissance du professionnel de santé, de tout membre du personnel de ces établissements ou organismes et de toute autre personne en relation, de par ses activités, avec ces établissements ou organismes. » (CSP, art. 1110-4.) Les patients experts y sont donc également soumis. Cela est confirmé par l'article 226-13 du code pénal : «La révélation d'une information à caractère secret par une personne en raison [...] d'une mission temporaire, est punie d'un an d'emprisonnement et 15000 euros d'amende. » Les établissements de santé pourront donc faire appel à des personnes atteintes d'une pathologie dans le cadre de séances collectives sans crainte que ceux-ci ne dévoilent certaines informations confidentielles dont ils auraient eu connaissance puisqu'ils sont soumis au même régime que les professionnels de santé.

Illustration : un patient éducateur se trouve être l'assureur d'un autre patient de son groupe. Tenu par le secret professionnel relatif à son activité au sein d'un établissement de santé, il ne pourra en aucun cas utiliser des informations sur ce patient dans son activité d'assureur. Mais dans le cadre du secret partagé, sous réserve que les autres patients ne s'y soient pas opposés, il pourra donc avoir accès à des données médicales si celles-ci se révèlent utiles pour sa mission d'éducation thérapeutique.

En pratique, il sera bien sûr beaucoup plus facile de limiter l'accès à certaines informations lorsque le dossier est informatisé plutôt qu'en libre accès sur papier.

D'une manière générale, que les non-professionnels soient informés de certaines données confidentielles ne pose en soit pas de problème puisqu'ils sont soumis au secret professionnel; en cas de divulgation d'une information ils encourent une sanction pénale (violation du secret professionnelle) et civile (dommages et intérêts).

Malgré ces dispositions, on constate une certaine réticence du monde médical sur la participation de non-professionnels à l'éducation thérapeutique en raison des problèmes liés à la confidentialité.

Pour davantage de sécurité juridique, il peut être envisagé de faire signer une clause de confidentialité à tous les nonsoignants qui seraient en contact avec des patients dans le cadre de séances collectives. C'est un engagement contractuel qui va définir quels sont les actes autorisés et interdits. Il devra préciser entre autre la durée de confidentialité. En cas de violation, le patient victime pourra agir sur le fondement de la responsabilité civile contractuelle pour non respect de la vie privée (article 9 du code civil) et ainsi obtenir des dommages et intérêts.

- La confidentialité. Voilà un autre aspect tout aussi important qui nécessite d'être abordé dans le cadre du groupe de patients lui-même. Dans l'exemple de l'assureur cité ci-dessus, il paraitt plus dangereux que celui-ci se trouve au sein du groupe de patients qu'au sein de l'équipe thérapeutique, car ce dernier cas lui confère des obligations telles que le respect du secret professionnel, régime très protecteur pour le patient; en revanche, ce type d'obligation n'existe pas entre patients et il importe donc de faire en sorte qu'ils puissent échanger librement sans crainte d'une divulgation à caractère personnel par la suite. Un établissement de santé voulant mettre en place des séances collectives devra ainsi rappeler en début de séance les règles inhérentes à respecter : discrétion, confidentialité 
des échanges, liberté de silence comme de parole, non jugement... Cette obligation de discrétion peut également figurer dans le document de consentement signé au départ sous la forme de principes généraux à respecter ou dans une charte expliquant les différents droits et obligations à la charge des professionnels mais aussi des patients.

Il est important également d'informer le groupe de patients sur ce qu'ils encourent en cas de divulgation d'une information à caractère confidentiel afin de les responsabiliser. En effet, la personne qui s'en estime victime pourra agir sur le fondement de la violation du respect de la vie privée énoncé à l'article 9 du code civil : «Chacun a droit au respect de sa vie privée. » Il revient au juge d'apprécier l'existence ou non d'une infraction en vertu de son pouvoir d'appréciation souveraine. La victime devra prouver une faute, un préjudice et un lien de causalité, et pourra alors obtenir des dommages et intérêts.

Une seconde voie, pénale cette fois, est ouverte pour la personne ayant subi ce type de préjudice. Il s'agit ici de démontrer l'élément moral, c'est-à-dire la volonté de nuire de la part de celui qui a divulgué l'information confidentielle et l'élément matériel.

«Est puni d'un an d'emprisonnement et de 45000 euros d'amende le fait, au moyen d'un procédé quelconque, volontairement de porter atteinte à l'intimité de la vie privée d'autrui :

1. En captant, enregistrant ou transmettant, sans le consentement de leur auteur, des paroles prononcées à titre privé ou confidentiel;

2. En fixant, enregistrant ou transmettant, sans le consentement de celle-ci, l'image d'une personne se trouvant dans un lieu privé. » (Art. 226-1 du code pénal.)

Par ailleurs, un autre aspect juridique nécessite une attention particulière : les responsabilités encourues par les professionnels qui mettent en place un programme aussi bien dans sa conception que dans son animation.

\section{La problématique de la responsabilité juridique en éducation thérapeutique du patient}

L'éducation thérapeutique fait partie intégrante des soins, dont elle n'est ni un préalable, ni un corollaire. De fait, et à défaut d'un régime juridique particulier décidé par le législateur ou reconnu par la jurisprudence, l'éducation thérapeutique est soumise au même régime juridique que tout acte de soins.

\subsection{Conception d' un programme d'ETP et mise en cause de sa responsabilité juridique}

Un programme peut être initié exclusivement par des professionnels de santé, co-initié par des professionnels et associations de patients ou personnes atteintes de la pathologie en question.
La question se pose de savoir si un patient victime d'un dommage peut agir sur le fondement de la mauvaise conception, mise en place d'un programme. Cette hypothèse semble très difficile à imaginer, dans la mesure où le programme est validé par l'agence régionale de santé (ARS) après une évaluation effectuée sur la base des recommandations de la HAS. La victime qui souhaite malgré tout agir, le fera sur le fondement de la responsabilité civile (d'après l'article 1382 du code civil) contre l'ARS, en démontrant une faute, un préjudice et un lien de causalité.

Une difficulté peut néanmoins être soulevée. Que se passerait-t-il si un programme approuvé par l'ARS ne correspond pas en pratique à ce qui a été validé ? Par exemple, si l'établissement ne dispense que 5 séances sur les 10 programmées, qui est responsable en cas de dommage - ce dernier pouvant consister pour un patient diabétique ne sachant pas reconnaître les signes d'une crise d'hypoglycémie et adapter les doses en conséquence à commettre une erreur et aggraver son état de santé ? L'ARS ne sera pas responsable puisqu'elle a validé un programme conforme, différent de celui qui a été mis en place, mais nous pouvons imaginer que celle-ci vérifiera la conformité ou non du programme qui fait l'objet d'un contentieux. Le patient victime, quant à lui, agira contre l'établissement en question, soit en responsabilité civile contractuelle si l'établissement est privé, soit en responsabilité administrative pour un établissement public.

\subsection{La responsabilité juridique liée à l'animation d'un programme d'ETP}

Avant de parler des fondements sur lesquels la responsabilité peut être engagée, il est nécessaire de s'interroger sur le cadre juridique à définir pour éviter des problèmes a posteriori.

Lorsque l'animation d'un programme est assurée exclusivement par des professionnels de santé, aucune contractualisation particulière n'est à respecter. En revanche, lorsqu'elle est assurée à la fois par des soignants et des membres associatifs ou un patient expert, il est indispensable de définir une coordination entre l'hôpital et ladite association. Une convention doit être conclue entre eux en vertu de l'article L1112-5 du code de santé publique : "Les associations qui organisent l'intervention des bénévoles dans des établissements de santé publics ou privés doivent conclure avec les établissements concernés une convention qui détermine les modalités de cette intervention», et selon la circulaire DHOS du 4 octobre 2004 relative à la convention définissant les conditions d'intervention des associations de bénévoles dans les établissements de santé.

Cette contractualisation entre les deux parties a pour but de garantir le statut de bénévole aux non-soignants (membres associatifs, patients experts), acte indispensable pour être couvert par l'assurance de l'établissement en cas de mise en œuvre de la responsabilité.

Il paraît important de mettre ce point en exergue dans le cas de professionnels faisant intervenir des patients experts rattachés à aucune association. Une pratique courante, alors que 
cela s'avère dangereux en termes de responsabilité, puisque ce non-soignant ne sera pas couvert par l'établissement en cas de faute. Dans ce cas, il convient absolument de prévenir l'assureur de l'établissement afin de couvrir cette personne. Faute de quoi elle sera responsable sur ses propres deniers.

Cette mise en œuvre d'une coordination est nécessaire pour définir les modalités de responsabilité.

En cas de préjudice subi par une personne, un établissement public engagera sa responsabilité administrative, un établissement privé sa responsabilité civile. Dans ces deux cas, les soignants, ainsi que les bénévoles associatifs ou patients experts sont couverts par l'établissement lorsqu'ils commettent une faute dans le cadre de leur activité de soins. C'est seulement dans l'hypothèse d'une faute personnelle en raison de son caractère intentionnel ou par sa particulière gravité que le soignant sera personnellement responsable sur le plan indemnitaire. La victime peut néanmoins agir contre l'établissement qui se retournera alors à son tour contre l'auteur du dommage.

Pour la responsabilité pénale, chaque soignant est personnellement responsable dans le cas de violation du secret professionnel ou de faute d'imprudence (art. 121-1 du code pénal).

\subsection{Les motifs juridiques en lien avec l'animation d'un programme d'ETP}

Plusieurs motifs peuvent être invoqués pour engager la responsabilité jurique de l'équipe au titre de son activité d'éducation thérapeutique :

- La violation du secret professionnel. L'auteur de l'infraction encourt un an de prison et 15000 euros d'amende. La charge de la preuve pèse sur le demandeur, le patient victime. L'auteur sera personnellement responsable non seulement sur le plan pénal mais aussi civil puisqu'il s'agit d'une faute personnelle. En ETP, cette hypothèse peut se rencontrer lorsqu'un soignant mais aussi un non-professionnel divulguent des informations à caractère confidentiel vues, entendues ou comprises au cours du diagnostic éducatif, ou lors des séances individuelles ou collectives. $C f$. art. 226-13 du code pénal.

- Le non-respect de la vie privée. La violation de l'intimité entraîne des conséquences civiles (dommages et intérêts). L'auteur de la violation sera responsable sur le plan civil. En ETP, si un des patients membre du groupe lors des séances collectives divulgue une information confidentielle, la victime pourra agir sur ce fondement ( $c f$. art. $9 \mathrm{du}$ code civil).

- Le devoir d'information paraît important en la matière. Il s'agit de transmettre au patient une information claire, loyale et adaptée. Pour que l'éducation thérapeutique produise ses effets, il est nécessaire de parvenir à transmettre un certain savoir sur la maladie et sur les soins, en veillant à la bonne compréhension des informations par le patient. La charge de la preuve pèse sur le professionnel qui devra démontrer le temps accordé à l'information et sur sa qualité, d'où la nécessité de la tracer sans tarder dans le dossier d'éducation (un dossier informatique renforce la qualité de la preuve) ( $c f$. art. 1111-1 du CSP). Le patient victime d'un incident chez lui à la suite d'un programme pourra engager la responsabilité de la structure pour manquement au devoir d'information, en évoquant le fait qu'elle n'a pas été assez précise et claire. La phase d'évaluation du patient est donc déterminante : plus elle sera effectuée de manière précise et plus les juges pourront déterminer facilement si le patient était titulaire d'une information suffisamment claire et adaptée.

- La faute non intentionnelle d'imprudence, négligence. «Il y a délit en cas de faute d'imprudence, négligence, s'il est établi que l'auteur n'a pas accompli les diligences normales compte tenu, de la nature de ses missions ou de ses fonctions, de ses compétences ainsi que du pouvoir et les moyens dont il disposait.» (Art. 121-3 du code pénal.) Et «les personnes physiques qui n'ont pas causé directement le dommage mais qui ont créé ou contribué à créer la situation et qui ont permis la réalisation du dommage sont responsables pénalement si elles ont commis une faute délibérée ou caractérisée. » Cet alinéa a été introduit à la suite de la loi de 2000; la responsabilité pénale d'une personne peut être recherchée alors même qu'elle a commis une faute non intentionnelle dès lors que celle-ci est délibérée ou caractérisée, c'est-à-dire lorsque l'auteur a conscience d'exposer la victime à un risque d'une particulière gravité et dont il était au courant.

Illustration : un patient rentre chez lui après un programme d'éducation thérapeutique mais sa négligence conduit à l'échec des soins et une aggravation irréversible de son état de santé. Il ne pourra prétendre à une indemnisation en l'absence de lien de causalité entre le comportement du médecin qui l'a pris en charge et le dommage subi. Toutefois, s'il démontre que le professionnel n'a pas causé directement le dommage mais contribué à créer la situation en commettant une faute délibérée ou caractérisée, il pourra agir sur l'article 121-3 du code pénal.

La phase d'évaluation est importante :

- le patient apte à être autonome et à gérer seul son traitement durant cette dernière ne pourra pas engager la responsabilité de l'équipe s'il commet une maladresse à son domicile;

- en revanche, il pourra demander réparation s'il démontre qu'elle savait à ce moment-là qu'il ne pouvait s'autoadministrer un traitement, et a donc créé ou contribué à créer la situation ayant entraîné le dommage.

Nous ne pouvons donc que conseiller aux équipes voulant se protéger au maximum du risque judiciaire d'élaborer des fiches d'évaluation d'une grande précision.

Si au moment de l'évaluation, le patient ne possède pas les compétences nécessaires, cela sera indiqué dans sa fiche ou sur son compte-rendu éducatif tenu après chaque séance. 


\section{Conclusion}

En matière d'éducation thérapeutique, les principales problématiques sur le plan juridique sont donc :

- La confidentialité : les différents intervenants et notamment le «patient expert» sont tenus par le secret professionnel. On précaunisera pour les établissements souhaitant une plus grande sécurité juridique la signature d'une clause de confidentialité pour les professionnels non soignants.

- La responsabilité juridique : différents motifs sont envisageables. Parmi eux, la faute non intentionnelle et le défaut d'information pour lesquels la phase d'évaluation des compétences est importante car : dans le premier cas, elle va permettre de déterminer si le patient était titulaire d'une information pertinente; dans le second, s'il était apte à être autonome - conditions qui mettent à l'abris les équipes du risque judiciaire.

Les soignants sont désormais soumis au respect d'obligations dans le cadre de la pratique de l'ETP. Mais ils sont encore peu nombreux à avoir conscience de ces nouvelles exigences et de la responsabilité juridique qui pèsent sur eux en raison de l'intégration récente dans le domaine législatif. L'ETP réactive un arsenal juridique qu'on avait un peu tendance à oublier. Si la jurisprudence est actuellement peu importante voire inexistante dans ce domaine, elle se développera immanquablement au cours des prochaines années.

Loin de vouloir freiner ou figer les nombreuses initiatives en ETP qui se mettent en place un peu partout, le rappel de la loi et des risques encourus doit au contraire permettre d'interroger les pratiques d'éducation et de soins et inciter à une grande rigueur dans leur application au regard du droit français et dans l'esprit de la loi du 4 mars 2002 où le droit du patient est clairement explicité.

Conflit d'intérêt : Aucun.

Remerciements. Pour leur contribution à la réalisation de cette étude à Cyril Crozet, Jean-Charles Verheye et Claire Marchand, membres du Laboratoire de Pédagogie de la Santé EA 3412 Université Paris 13, Bobigny.

\section{Références}

1. Fournier C, Buttet P, David M, Périgois E., Éducation du patient dans les établissements de santé français : l'enquête ÉDUPEF. Evolutions 2008; 9: 1-6.
2. Code de la santé publique, Première partie : Protection générale de la santé, Livre Ier: Protection des personnes en matière de santé, titre VI : Éducation thérapeutique du patient. [consulté le 16 décembre 2010] ; disponible à l'adresse suivante : http://www.legifrance.gouv.fr/affichCode. do? cidTexte $=$ LEGITEXT000006072665 $\&$ dateTexte $=20110210$

3. Organisation Mondiale de la Santé. Therapeutic patient education: continuing education programmes for health care providers in the field of prevention of chronic diseases: report of a WHO working group. Copenhagen: WHO Regional office for Europe; 1998.

4. Haute Autorité de Santé. Éducation thérapeutique du patient : définition, finalités et organisation. Juin 2007 [consulté le 16 décembre 2010] ; disponible à l'adresse suivante : www.has-sante.fr/portail/upload/docs/application/pdf/etp_ -_definition_finalites_-_recommandations_juin_2007.pdf

5. Guérin G, Prise en charge et protection sociale, éducation thérapeutique des personnes atteintes de maladies chroniques : deux rapports du HCSP. ADSP 2010; 72:45-47.

6. Gagnayre R, d'Ivernois J-F, Apprendre à éduquer le patient : approche pédagogique. $4^{\mathrm{e}}$ ed. Coll. "Éducation du patient", Paris : Maloine ; 2010.

7. Bury J-A, Éducation pour la santé, concepts, enjeux, planifications. Coll. Savoir \& Santé. Questions, Bruxelles : De Boeck ; 1988.

8. Cour de Cassation, chambre Criminelle, 15 mai 1984, Amzallag D. 1986, p. 106, note G. Mémeteau.

9. CJCE, 31 janvier 1984, Luisi et Carbone, 286/82, point $n^{\circ} 16$, Rec. CE, p. 377.

10. Van Ballekom K, L'éducation du patient en hôpital : l'autonomie du patient : de l'information à l'éducation. Godinne : Centre d'Education du Patient asbl ; 2008. [consulté le 16 décembre 2010] ; disponible à l'adresse suivante : http://www.educationdupatient.be/cep/pdf/dossiers/ education_du_patient_en_hopital_fr.pdf

11. Expert Patients Programme Courses for Long Term Health Conditions [Site Internet]. 2007 [consulté le 16 décembre 2010] ; disponible à l'adresse suivante : http://www.expertpatients.co.uk/

12. Reach G. Une critique du concept de patient éducateur. Médecine des maladies métaboliques 2009; 3(1):89-94.

13. Wintz L., Sannié T., Ayçaguer S, Guercois S, Bernhard JP, Valluet $\mathrm{D}$, et al., Patient resources in the therapeutic éducation of haemophiliacs in France: their skills and roles as defined by consensus of a working group, Haemophilia 2010; 16:447-454. 